\title{
Benzene poisoning presenting as status epilepticus: a case report and literature review
}

\author{
Di Wang^, Xiaogen Tao^ \\ Department of Intensive Care Medicine, The First Affiliated Hospital of USTC, Division of Life Sciences and Medicine, University of Science and \\ Technology of China, Hefei, China \\ Correspondence to: Xiaogen Tao. Department of Intensive Care Medicine, The First Affiliated Hospital of the University of Science and Technology of \\ China, No. 17 Lujiang Road, Hefei 230036, China. Email: txg0724@163.com.
}

\begin{abstract}
Benzene is a common industrial chemical and an important environmental pollutant. In addition, exposure to benzene may cause injury to the nervous system, in vivo. However, few clinical cases of benzene-induced injury to the nervous system have been reported. Therefore, the present report highlights a case of benzene poisoning, presenting as status epilepticus. The patient was admitted to the intensive care unit (ICU) with a coma after experiencing seizures 7 hours ago. He had a history of exposure to paint containing benzene. In addition, cranial magnetic resonance imaging (MRI) revealed extensive bilateral signal abnormalities in the cerebral white matter. The level of the benzene metabolite was also high in the urine. Consequently, the patient was diagnosed with benzene poisoning and status epilepticus, after which he received nerve nourishment, enteral nutrition, mechanical ventilation, and other supportive measures. He regained normal consciousness and motor ability, 1 month after treatment. The patient was also followed-up for 15 months and it was shown that he had returned to normal life without neurological and psychological deficits. Moreover, cranial MRI showed that the lesions had disappeared. This case therefore indicated that benzene poisoning should be considered if the patient has a clear history of exposure to the chemical, presents with seizures and has extensive signal abnormalities in the white matter, revealed by MRI examination. Additionally, early diagnosis and effective supportive treatment can guarantee a favorable prognosis for benzene poisoning.
\end{abstract}

Keywords: Benzene poisoning; epileptic seizures; urine phenol; cranial magnetic resonance imaging (MRI); case report

Submitted Apr 08, 2021. Accepted for publication Jul 20, 2021.

doi: 10.21037/atm-21-1726

View this article at: https://dx.doi.org/10.21037/atm-21-1726

\section{Introduction}

Benzene is a commercial chemical compound that is used in a wide range of applications, including plastics, synthetic fibers, dyes, drugs, and detergents. Additionally, benzene is highly carcinogenic and exposure to the chemical leads to an increased risk for hematological malignancy, including the myelodysplastic syndrome, aplastic anemia and acute myeloid leukemia (1). Mechanically, benzene induces oxidative stress, DNA lesions and epigenetic alterations, leading to cytotoxicity and hematotoxicity (2). Benzene also causes chronic inflammation and immunosuppression by activating the innate immune system and suppressing the adaptive immune system (3). Moreover, a recent study showed that benzene could damage cerebellar structure and function in rats (4). However, very few cases of benzeneinduced injury to the nervous system, have been reported.

^ ORCID: Di Wang, 0000-0002-8279-0346; Xiaogen Tao, 0000-0002-0959-8876. 
Consequently, the present report highlights a case of status epilepticus caused by benzene poisoning, with a complete clinical profile, especially characteristic MRI findings on admission and the outcome of follow-up after 15 months. This case indicates that benzene poisoning should be considered if the patient has a history of benzene exposure and presents with epileptic seizures. In addition, early diagnosis and prompt treatment can confer a favorable prognosis for benzene poisoning. We present the following article in accordance with the CARE reporting checklist (available at https://dx.doi.org/10.21037/atm-21-1726).

\section{Case presentation}

All procedures performed in studies involving human participants were in accordance with the ethical standards of the institutional and/or national research committee(s) and with the Helsinki Declaration (as revised in 2013). Written informed consent was obtained from the patient.

A 32-year-old man was admitted to the emergency department of The First Affiliated Hospital of the University of Science and Technology of China with a coma after experiencing seizures 7 hours ago. He had experienced fatigue and poor appetite a few days before admission. Seven hours before admission, he presented with five continuous episodes of generalized tonic-clonic seizures, each lasting a few minutes, then finally became comatose. In addition, he had worked for about 1 month in a printing factory located in the rural area of Anhui Province, China. He had no past medical or traumatic history except for chronic hepatitis B infection which was being treated using tenofovir, for the past 7 years. Due to the frequent generalized seizures, he was intubated then admitted to the intensive care unit (ICU).

Physical examination revealed unconsciousness, a GCS score of $5 \mathrm{~T}$ (E1, VT, M3), a bilateral pupil diameter of $5 \mathrm{~mm}$, a normal pupillary light reflex and a positive Babinski sign. Additionally, examination of the cerebrospinal fluid (CSF) revealed normal appearance, nucleated cells 1 cell/ $\mu \mathrm{L}$, a slightly elevated glucose level of $5.04 \mathrm{mmol} / \mathrm{L}$ and a normal protein level of $0.3 \mathrm{~g} / \mathrm{L}$. Except for the positive serological test for hepatitis $\mathrm{B}$, all the causative pathogens tests were negative, including a next-generation sequencing (NGS) test of the CSF. Moreover, cranial MRI showed that high signal intensities were symmetrically distributed in the cerebellar dentate nucleus, basal ganglia and cerebral white matter in the T1-weighted FLAIR, T2-weighted PROPELLER and diffusion-weighted imaging (DWI) scans (Figure 1). He was initially diagnosed with status epilepticus and suspected viral encephalitis. Therefore, pharmacologic treatment included acyclovir for empirical antiviral therapy, propofol for sedation and sodium valproate along with phenobarbital for epilepsy. Furthermore, supportive measures included airway management, mechanical ventilation and maintenance of the electrolyte and acid-base balance.

Considering that his workplace was a printing factory, a toxicology test on his urine was ordered from Anhui Prevention and Treatment Center for Occupational Disease. The results showed increased urine concentration $(62 \mathrm{mg} / \mathrm{L})$ of phenol which is the major metabolite of benzene. Although no workplace sampling was obtained to test the presence of benzene, his history of occupational exposure to benzene was further confirmed by the fact that he was constantly in contact with paint containing benzene, and he would work without personal protective equipment. Therefore, he was finally diagnosed with benzene poisoning and status epilepticus. Consequently, treatment with acyclovir was stopped and the patient was given nerve nourishment, enteral nutrition, and other supportive measures. Notably, his GCS scores improved to 8T (E4, VT, M3) after 10 days of treatment in the ICU. He was then transferred to the neurology department with normal consciousness and motor ability, after 27 days of admission in the ICU. Furthermore, 15 months of follow-up revealed that he had returned to normal life without any neurological and psychological deficits and cranial MRI showed that the lesions had disappeared (Figure 2). Timeline of diagnosis, treatment, and follow-up of the patient shown in Figure 3.

\section{Discussion}

Benzene is one of the most toxic air pollutants in the environment. Epidemiologic and experimental research also shows that exposure to benzene can affect vital organs and systems in the body, such as the respiratory, reproductive, nervous, immune, endocrine and cardiovascular systems (5). In addition, hematological diseases induced by chronic exposure to benzene have been widely studied (1). However, few clinical cases of neurological damage induced by benzene exposure have been reported.

A recent study reported a case of cognitive impairment induced by chronic exposure to benzene (6). The patient was a painter with occupational exposure to benzene and had suffered recent memory loss, cognitive impairment, and autonomic nerve dysfunction. Additionally, MRI showed abnormal symmetric signals in the temporal lobe, frontal 

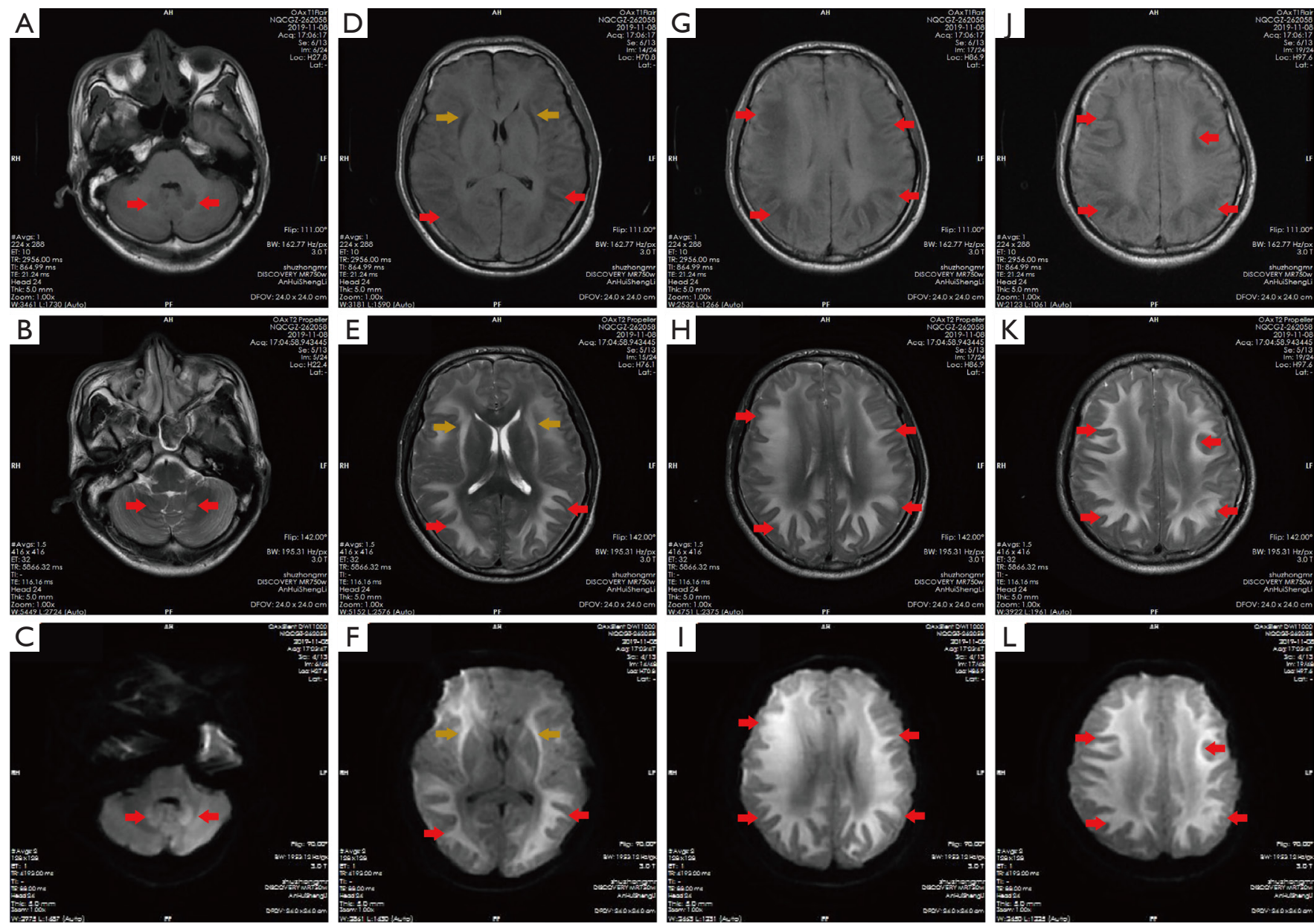

Figure 1 (A-L) show cranial MRI images of the patient on admission. The T1-weighted FLAIR, T2-weighted PROPELLER and DWI scans show high signal intensities at (A-C) the cerebellar dentate nucleus (red arrow); (D-F) basal ganglia (yellow arrow); and (D-L) cerebral white matter (red arrow). MRI, magnetic resonance imaging; DWI, diffusion-weighted imaging.

lobe, occipital lobe, internal capsule, and the basal ganglia. Examination of the CSF was also negative for antibodies related to autoimmune encephalitis. The patient was subsequently diagnosed with cognitive decline induced by chronic benzene poisoning, after which she received nerve nourishment, anti-inflammatory treatment, hyperbaric oxygen, nutritional support, and other supportive treatments. She showed notable improvement in cognitive function 1 month after treatment and her cognitive function had almost returned to normal, 3 months following discharge.

To the best of our knowledge, this is the first case of benzene poisoning presenting with status epilepticus. The patient had a clear history of contact with benzene; however, no workplace sampling was obtained to confirm the presence of benzene, which is the limitation of this case. The presence of epileptic seizures and cranial MRI findings revealed injury of the central nervous system, caused by benzene. Notably, the cranial MRI findings in the present case were essentially consistent with those from the previously reported case of chronic benzene poisoning. This suggests that damage to the cerebral white matter is a critical pathogenic process of benzene poisoning. Moreover, characteristic MRI findings provide useful clues for the diagnosis of benzene-induced injury of the central nervous system. Therefore, based on this case and the one previously reported, the clinical characteristics of benzene-induced injury of the central nervous system can be summarized as follows; definite exposure to benzene, symptoms of nervous system injury, including cognitive impairment and seizures, extensive signal abnormalities in the white matter, revealed 

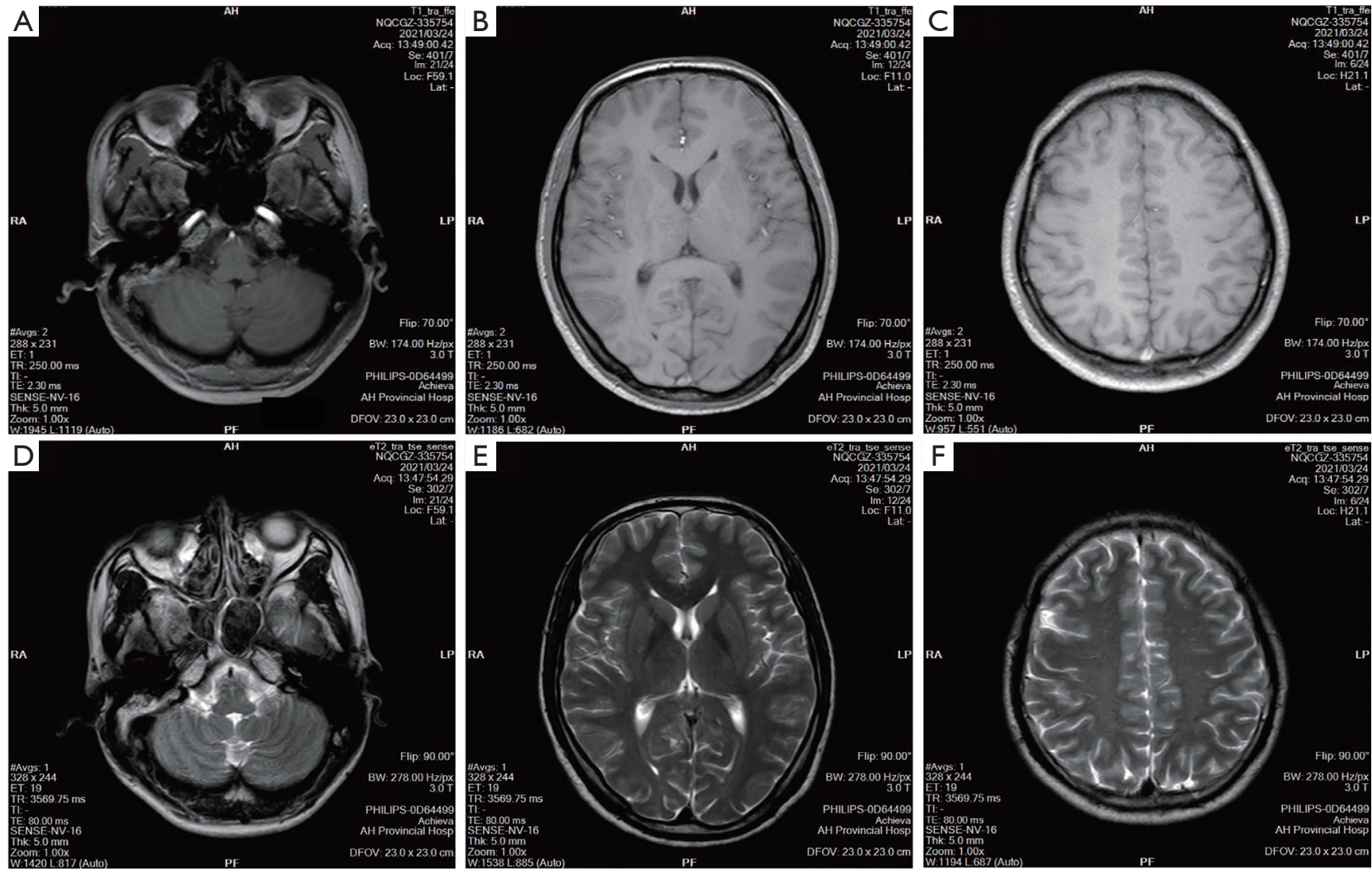

Figure 2 Cranial MRI images of the patient after 15 months of follow-up show normal signal intensity at (A-C) T1-weighted; and (D-F) T2-weighted scans. MRI, magnetic resonance imaging.

At the emergency

- department

- Chief complaints: a coma for seven hours after seizures.

- The patient was intubated and then admitted to the ICU.
In the neurology

$\longrightarrow$ department

- The patient was transferred to the neurology department with normal consciousness and motor ability.

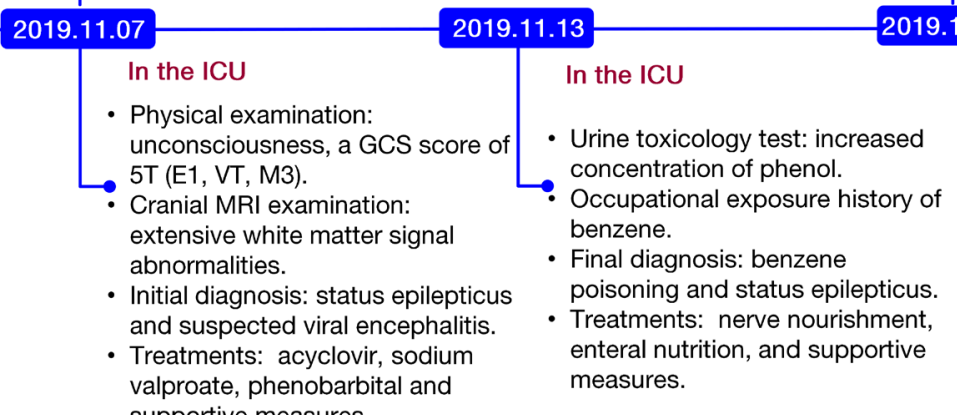
2021.03.24

Figure 3 Timeline of diagnosis, treatment, and follow-up of patient. ICU, intensive care unit; MRI, magnetic resonance imaging. 
by MRI examination and favorable prognosis after timely and effective treatment.

Furthermore, little information exists on the mechanisms of the benzene-induced injury to the nervous system. A previous in vivo study showed that rats treated with benzene presented with anxiety and motor impairment. Additionally, treatment with benzene resulted in a $34 \%$ decrease in the total volume of the cerebellar hemisphere, including the medulla and cortex. There was also a significant decrease in the amount of Purkinje neurons, Bergmann glial cells, Golgi cells, granule cells as well as neurons and glial cells in both the molecular layer and the intracerebellar nuclei (4). Another study showed that exposure to benzene induced hypothalamic inflammation in mice by increasing the amount of microglial cells in the medial basal hypothalamus and the expression levels of inflammatory genes in the arcuate nucleus (7).

In conclusion, to best of our knowledge, this is the first case report of benzene poisoning presenting with status epilepticus. Therefore, benzene poisoning should be considered if the patient has a clear history of exposure to the chemical, presents with seizures and has extensive signal abnormalities in the white matter, revealed by MRI examination. Moreover, early diagnosis and effective supportive treatment can guarantee a favorable prognosis for benzene poisoning.

\section{Acknowledgments}

Funding: None.

\section{Footnote}

Reporting Checklist: The authors have completed the CARE reporting checklist. Available at https://dx.doi. org/10.21037/atm-21-1726

Conflicts of Interest: Both authors have completed the ICMJE uniform disclosure form (available at https://dx.doi. org/10.21037/atm-21-1726). The authors have no conflicts of interest to declare.

Ethical Statement: The authors are accountable for all aspects of the work in ensuring that questions related to the accuracy or integrity of any part of the work are appropriately investigated and resolved. All procedures performed in studies involving human participants were in accordance with the ethical standards of the institutional and/or national research committee(s) and with the Helsinki Declaration (as revised in 2013). Written informed consent was obtained from the patient for publication of this manuscript and any accompanying images.

Open Access Statement: This is an Open Access article distributed in accordance with the Creative Commons Attribution-NonCommercial-NoDerivs 4.0 International License (CC BY-NC-ND 4.0), which permits the noncommercial replication and distribution of the article with the strict proviso that no changes or edits are made and the original work is properly cited (including links to both the formal publication through the relevant DOI and the license). See: https://creativecommons.org/licenses/by-nc-nd/4.0/.

\section{References}

1. Gross SA, Paustenbach DJ. Shanghai Health Study (20012009): What was learned about benzene health effects? Crit Rev Toxicol 2018;48:217-51.

2. McHale CM, Zhang L, Smith MT. Current understanding of the mechanism of benzene-induced leukemia in humans: implications for risk assessment. Carcinogenesis 2012;33:240-52.

3. Guo H, Ahn S, Zhang L. Benzene-associated immunosuppression and chronic inflammation in humans: a systematic review. Occup Environ Med 2021;78:377-84.

4. Rafati A, Erfanizadeh M, Noorafshan A, et al. Effect of benzene on the cerebellar structure and behavioral characteristics in rats. Asian Pac J Trop Biomed 2015;5:568-73.

5. Bahadar H, Mostafalou S, Abdollahi M. Current understandings and perspectives on non-cancer health effects of benzene: a global concern. Toxicol Appl Pharmacol 2014;276:83-94.

6. Hu J, Yu E, Liao Z. Changes in cognitive function and related brain regions in chronic benzene poisoning: a case report. Ann Transl Med 2021;9:81.

7. Debarba LK, Mulka A, Lima JBM, et al. Acarbose protects from central and peripheral metabolic imbalance induced by benzene exposure. Brain Behav Immun 2020;89:87-99.

Cite this article as: Wang D, Tao X. Benzene poisoning presenting as status epilepticus: a case report and literature review. Ann Transl Med 2021;9(15):1263. doi: 10.21037/atm21-1726 